\title{
On the phenomenology of sphaleron-induced processes at the LHC and beyond
}

\author{
Andreas Papaefstathiou, ${ }^{a, b}$ Simon Plätzer ${ }^{c}$ and Kazuki Sakurai ${ }^{d}$ \\ ${ }^{a}$ Institute for Theoretical Physics Amsterdam and Delta Institute for Theoretical Physics, \\ University of Amsterdam, Science Park 904, 1098 XH Amsterdam, The Netherlands \\ ${ }^{b}$ Nikhef, Theory Group, \\ Science Park 105, 1098 XG, Amsterdam, The Netherlands \\ ${ }^{c}$ Particle Physics, Faculty of Physics, University of Vienna, \\ Vienna, Austria \\ ${ }^{d}$ Institute of Theoretical Physics, Faculty of Physics, University of Warsaw, \\ ul. Pasteura 5, PL-02-093 Warsaw, Poland \\ E-mail: apapaefs@cern.ch, simon.plaetzer@univie.ac.at, \\ kazuki.sakurai@fuw.edu.pl
}

ABSTRACT: We investigate the phenomenological aspects of non-perturbative baryon- and lepton-number-violating processes at hadron colliders. Such processes, induced by instanton/sphaleron configurations of the electroweak gauge fields, are believed to play a crucial role in the generation of baryon asymmetry in the early Universe at finite temperature. On the other hand, at colliders (that represent the zero-temperature high-energy regime) the rate and observability of such processes are still under debate. Motivated by current theoretical considerations, we construct a modern event generator within the general-purpose Herwig Monte Carlo framework, that aims to capture the most relevant features of the dominant processes. We perform a detailed phenomenological analysis focussing on the Large Hadron Collider, at $13 \mathrm{TeV}$ proton-proton centre-of-mass energy, a potential highenergy upgrade at $27 \mathrm{TeV}$ and the proposed Future Circular Collider (FCC-hh) at $100 \mathrm{TeV}$. We derive constraints on the expected rates for various parametrisations of our model. We find that all three colliders are capable of providing meaningful information on the nature of instanton/sphaleron-induced processes at various energy scales.

KEYwords: Phenomenological Models

ArXiv EPrint: 1910.04761 


\section{Contents}

1 Introduction 1

2 Monte Carlo simulations of sphaleron-induced processes 3

2.1 Process generation 4

2.2 Phase space generation and integration $\quad 5$

2.3 Leading-order matrix element, unitarity and boson distribution 5

$\begin{array}{ll}2.4 & \text { Parametrisation of boson distributions }\end{array}$

2.5 Multi-boson $B$ - and $L$-conserving processes 8

3 Phenomenology at hadron colliders $\quad 8$

3.1 Reconstructed distributions 9

$\begin{array}{lll}3.2 & \text { Projected sensitivities } & 15\end{array}$

$\begin{array}{lll}4 & \text { Conclusions } & 19\end{array}$

\section{Introduction}

Discerning the details of dynamics of baryon-number violation would be a crucial step towards an ab initio understanding of the observed baryon asymmetry of the Universe. In particular, a class of baryon-number-changing processes associated with electroweak theory has been long studied [1]. The computation of amplitudes for such transitions employs approximate classical solutions of the electroweak theory, known as instantons. At zero temperature, with zero energy, the amplitudes for such tunnelling processes can be estimated to be of order $\exp \left[-2 \pi / \alpha_{w}\right] \sim \mathcal{O}\left(10^{-82}\right)$, where $\alpha_{w} \sim 1 / 30$ is the $\mathrm{SU}(2)$ coupling constant. Evidently this would have rendered the processes in question phenomenologically irrelevant and this article particularly short.

Nevertheless per aspera ad astra,${ }^{1}$ and a number of subsequent calculations (see e.g. [38]) have shown that the rate of instanton-induced processes exponentially grows with energy $E$, albeit in the limit $E \ll E_{0}$, where $E_{0}$ is the energy scale at which the instanton approximation itself breaks down. More specifically, $E_{0}$ is the height of the barrier that separates sectors of the electroweak vacuum, characterised by different values of the so-called ChernSimons (or winding) number, $N_{\mathrm{CS}}$, as demonstrated schematically in figure 1. Furthermore, there exist static solutions of the classical equations of motion that are unstable and sit on top of the barrier. These are the so-called "sphalerons". The existence of these solutions allows a transition from one EW vacuum to another dynamically, going over the barrier with energies larger than $E_{0}$, as schematically illustrated in figure 1. Unlike instantons, 


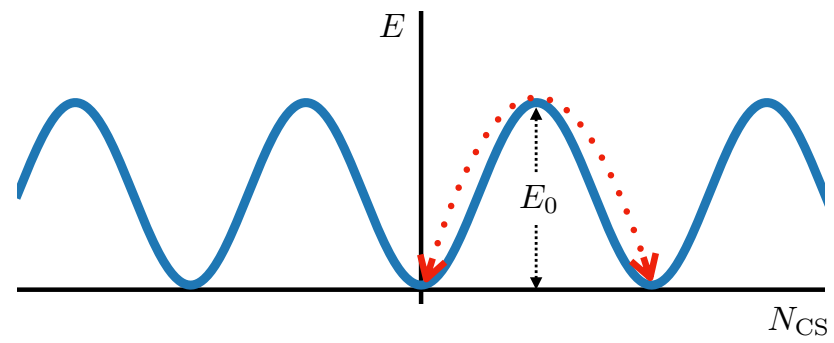

Figure 1. A schematic diagram of the degenerate Chern-Simons vacua, separated by a barrier of energy $E_{0}$. The result of a sphaleron transition between two of these vacua is illustrated.

this type of Chern-Simons number changing processes is not quantum tunneling, and thus not necessarily exponentially suppressed.

There have been several attempts to estimate the rate of the instanton/sphaleroninduced $\mathrm{EW}$ vacuum transition processes in the high-energy regime $\left(E \gtrsim E_{0}\right)$. For example, studies exploiting a semiclassical approximation concluded that the exponential suppression persists even at energy higher than $250 \mathrm{TeV}$ [9]. As was pointed out in the seminal paper by Klinkhamer and Manton [10], this may be due to a "few-to-many" suppression, which stems from a necessity, and difficulty, of assembling (highly-coherent and extended) instanton/sphaleron configurations from ordinary two-particle states in the collision. $^{2}$ On the other hand, other estimations based on the optical theorem suggest that the EW vacuum transition rate may become unsuppressed at energies around or above $20 \mathrm{TeV}[12,13]$. As pointed out in [14], the aforementioned few-to-many suppression may not be present because emitting one virtual gauge boson contributes a factor $g^{-1}$ to the amplitude in the instanton background, rather than $g$ as in the perturbative vacuum, and many gauge bosons can relatively easily be produced and assembled into a coherent state. A more recent study pointed out that it may be important to take the periodicity of the EW potential (see figure 1) into account, since the vacuum transition rate can be enhanced due to the resonant tunneling effect. They have estimated the EW vacuum transition rate by analysing the band structure of the spectrum and concluded that the instanton/sphaleron processes may become observably large at energies around or above $9 \mathrm{TeV}[14,15]$. Motivated by these encouraging estimates, several phenomenological studies on the zero-temperature instanton/sphaleron-induced processes have been carried out recently [16-23].

Although the potential for observing instanton/sphaleron-induced processes at colliders is not theoretically clear, one can instead turn to experiments to address the issue. Compared to the large uncertainty on the event rate, the signatures of such processes are relatively well understood. Due to the coupling of the fermions to the $\mathrm{SU}(2)$ gauge fields and the presence of the anomalous divergence of the axial-vector current, a change in $N_{\mathrm{CS}}$ implies a change in baryon $(B)$ and lepton $(L)$ numbers $\Delta L_{e}=\Delta L_{\mu}=\Delta L_{\tau}=\frac{1}{3} \Delta B=\Delta N_{\mathrm{CS}}$ as in figure 2. The enhancement is expected to occur when the process involves a large

\footnotetext{
${ }^{1}$ Latin, "Through hardships to the stars" [2].

${ }^{2}$ See also [11].
} 


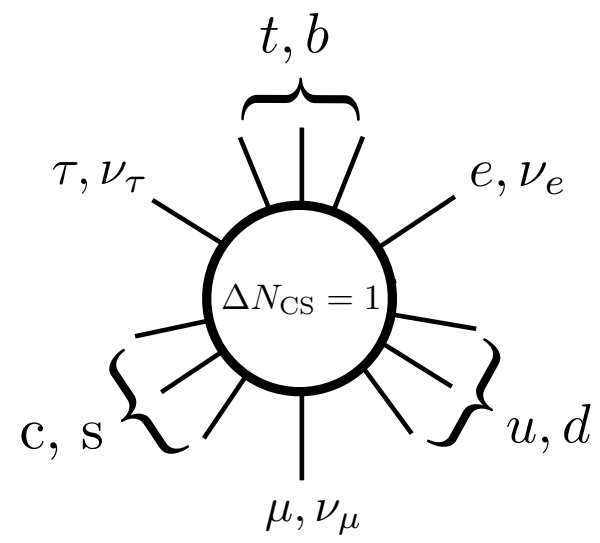

Figure 2. A schematic diagram of flavour structure of the process related to a unit change in Chern-Simons number, $\Delta N_{\mathrm{CS}}=1$.

number $\left(\sim 1 / \alpha_{w}\right)$ of gauge and/or Higgs bosons. ${ }^{3}$ The basic process would involve 12 left-handed fermions: 3 quarks for each generation and one lepton for each generation (figure 2). Hence, processes that could be observable at hadron colliders, schematically, would be of the form:

$$
q+q \rightarrow 7 \bar{q}+3 \bar{\ell}+n_{B} W / Z / \gamma / H,
$$

where $q, \bar{q}, \bar{\ell}$ denote a quarks, anti-quarks and anti-leptons, respectively, and $n_{B}$ is the total number of gauge and Higgs bosons. The charge structure of this process is explained in detail in the next section. From here on, we will refer to such processes as being "sphaleron" induced, emphasising the phenomenological nature of our analysis. We consider various parametrisations of the distributions of gauge bosons. We note, however, that we neglect the helicity of the produced fermions in our Monte Carlo (MC) simulation. We expect this to have a negligible effect on angular distributions that would be washed out by hadronization and other effects. ${ }^{4}$

The paper is organised as follows: we describe the MC simulation of sphaleron-induced processes in section 2. There, we also present a discussion of the uncertainties present in our parametrisation. In section 3 we present a study of the phenomenology of the processes at hadron colliders such as the CERN LHC at centre-of-mass energy of $13 \mathrm{TeV}$, a potential future upgrade to $27 \mathrm{TeV}$ and a potential Future Circular Collider colliding protons at $100 \mathrm{TeV}$.

\section{Monte Carlo simulations of sphaleron-induced processes}

We begin by describing the details of the MC event generator for sphaleron-induced processes that we have built, including the flavour structure, colour factors and the generation

\footnotetext{
${ }^{3}$ The relation between the change in the fermion and Chern-Simons numbers, as well as the enhancement associated with the large number of bosons, are illustrated rather neatly in the context of the twodimensional Abelian Higgs model, see e.g. [5, 6].

${ }^{4}$ Indeed, it is already challenging to determine the helicity of top quarks, that do not hadronize, even in processes that are less populated, see e.g. [24].
} 


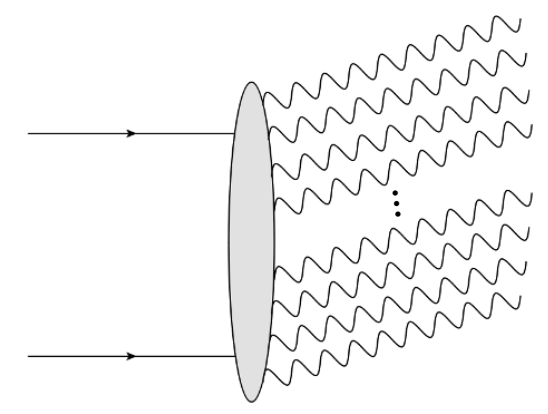

Figure 3. A schematic diagram of a $2 \rightarrow n$ processes that can be simulated via the "blob" matrix elements within the Herwig general-purpose event generator.

of the phase space. We also discuss the cross section in the context of unitarity and the parametrisation of the distribution of the number of gauge bosons.

\subsection{Process generation}

To facilitate the generation of $2 \rightarrow n$ processes in all generality, we have constructed customised infrastructure within the general-purpose Herwig Monte Carlo event generator [25-30]. This allows for the generation of "blob" matrix elements (MEs) through the definition of any $2 \rightarrow n$ process that can be viewed as a contact interaction. ${ }^{5}$ An example of such a process is shown in figure 3. We note that Herwig possesses the necessary infrastructure to handle the $\mathrm{SU}(3)$ colour source and sinks that appear in the $B$-violating processes [39]. We defer the description of the technical details to a future release note of Herwig.

For the simulation of sphaleron-induced processes, we only include the dominant $q q$ initial state given by eq. (1.1), where two valence quarks collide. The conjugate process is suppressed in the $p p$ collider due to a small ratio of the luminosity functions between valence-quark $(q q)$ and sea-quark $(\bar{q} \bar{q})$ initial states at $\sqrt{\hat{s}} \gtrsim \mathcal{O}(10) \mathrm{TeV}$. We take the initialstate partons to consist only of the light quarks $u$ and $d$ and we allow all quark generations in the produced final states, including the top and bottom quarks. The quark and lepton content of the process can be thought of as originating in a class of operators of the form

$$
\mathcal{O}_{\not B L} \sim\left(Q_{1} Q_{2} Q_{3}\right)\left(Q_{1}^{\prime} Q_{2}^{\prime} Q_{3}^{\prime}\right)\left(Q_{1}^{\prime \prime} Q_{2}^{\prime \prime} Q_{3}^{\prime \prime}\right) L^{1} L^{2} L^{3},
$$

where $Q$ and $L$ are the left-handed quark and lepton doublets, respectively. The anomaly argument suggests that all the degrees of freedom that are orthogonal to the $\mathrm{SU}(2)_{L}$ gauge group, i.e. colour and flavour, must appear at least and only once. With this condition, the colour indices of the quark fields must be constructed properly, making three "baryonic" (anti-symmetric) colour singlet combinations $(Q Q Q)$. Furthermore, the $\mathrm{SU}(2)_{L}$ indices of all quarks and leptons must be constructed in such a way that the operator is a $\mathrm{SU}(2)_{L}$ singlet. In terms of the left-handed doublet components, i.e. $(u, d)_{L}$ and $(\ell, \nu)_{L}$, this condition is equivalent to requiring the net electro-magnetic charge of the above operators

\footnotetext{
${ }^{5}$ This functionality should be particularly useful in the simulation of final states such as QCD instantons (e.g. [31-33]), microscopic black holes (e.g. [34-37]) and other non-perturbative multi-particle processes [38].
} 
is zero. ${ }^{6}$ In the hard process we generate colour flows compatible with this pattern on an equally likely, random basis. ${ }^{7}$

To be concrete, e.g. the combination $(u c t)(d s b)(u c t) e \mu \tau$ would form an allowed operator, but the combinations $(u d t)(c s b)(u c t) e \mu \nu_{\tau},(u u t)(d s b)(u c t) e \mu \nu_{\tau}$ or $(u c t)(d s b)(u c t) e e \nu_{\tau}$ would not be allowed, each violating one of the aforementioned conditions. In the event generation, we take into account all the possible allowed permutations of the quark flavours within the operator, that would lead to identical flavour content in the final state, by multiplying by an appropriate combinatorial "colour" factor. In other words, e.g. the final state $u d \rightarrow \bar{c} \bar{t} \bar{d} \bar{s} \bar{b} \bar{u} \bar{c} \bar{t} e^{+} \mu^{+} \bar{\nu}_{\tau}$ appears only once in the Herwig process generation, with its weight multiplied by the appropriate combinatorial colour factor. Explicitly, e.g. in the case of the $u d \rightarrow \bar{c} \bar{t} \bar{d} \bar{s} \bar{b} \bar{u} \bar{c} \bar{t} e^{+} \mu^{+} \bar{\nu}_{\tau}$ process, this factor is given by $[1 /(3 ! / 2 !)] \times(3 ! / 2 !)^{3}=9$, where the first factor takes into account that two of the first generation quarks have to be chosen to form the initial state and there is a factor of $(3 ! / 2 !)$ for each of the generations. At the stage of MC event generation, the colour configuration which determines which combinations of quarks form the colour singlets is chosen uniformly at random from the allowed ones.

\subsection{Phase space generation and integration}

Due to the lack of a detailed calculation describing the non-perturbative sphaleron-induced processes differentially, we distribute the particles uniformly in the available phase space. Two methods are particularly suited to the sampling of "flat" phase space at high multiplicities: the RAMBO [40] algorithm as described in [41], and the MAMBO algorithm according to [42]. The former was found to be inefficient for large numbers of massive particles, ${ }^{8}$ and therefore for all the phenomenological studies of the present article we have employed the MAMBO algorithm. Given the structure of the cross section, a detailed adaptive Monte Carlo integration is not required and we choose to predetermine a constant cross section value to select processes of equal final-state multiplicity. This further speeds up the phase space generation and integration. A sequence of different phase space generators might be considered in the future in order to attach definitive decays, or initial state splittings attached to the "blob" matrix element's legs.

\subsection{Leading-order matrix element, unitarity and boson distribution}

Our estimation for the boson multiplicity is based on the cross section formula from the leading-order matrix element (LOME) approach [3, 4, 43],

$$
\begin{aligned}
\hat{\sigma}_{\mathrm{LOME}}\left(\bar{n}_{B}, \bar{n}_{H}\right)= & C \mathcal{G}^{2} 2^{n_{B}} v^{-2 n}\left[\frac{\Gamma(n+103 / 12)}{\Gamma(103 / 12)}\right]^{2} \frac{1}{\bar{n}_{B} ! \bar{n}_{H} !} \\
& \times \int \prod_{i=1}^{10} \frac{d^{3} p_{i} E_{i}}{(2 \pi)^{3} 2 E_{i}} \prod_{j=1}^{\bar{n}_{B}} \frac{d^{3} p_{j}}{(2 \pi)^{3} 2 E_{j}} \frac{2\left(4 E_{j}^{2}-m_{W}^{2}\right)}{m_{W}^{2}} \times \prod_{k=1}^{n_{H}} \frac{d^{3} p_{k}}{(2 \pi)^{3} 2 E_{k}} \\
& \times(2 \pi)^{4} \delta^{4}\left(P_{\mathrm{in}}-\sum_{i=1}^{10} p_{i}-\sum_{j=1}^{\bar{n}_{B}} p_{j}-\sum_{k=1}^{\bar{n}_{H}} p_{k}\right),
\end{aligned}
$$

\footnotetext{
${ }^{6}$ See the original article by t'Hooft [1] or e.g. [6] for further details.

${ }^{7}$ Future work, however, should investigate this issue in more detail, specifically in presence of instantoninduced processes with a large number of additional gluons.

${ }^{8}$ The low efficiency was in fact the motivation for the MAMBO algorithm of [42].
} 
where $\bar{n}_{B}$ is the number of electroweak (EW) gauge bosons $(W, Z, \gamma), \bar{n}_{H}$ is the number of Higgs bosons in the final state. Also, $n_{B} \equiv \bar{n}_{B}+\bar{n}_{H}, n=n_{B}+10$ is the total number of final state particles, $v$ represents the VEV of the Higgs field, $\mathcal{G} \equiv 1.6 \cdot 10^{-101} \mathrm{GeV}^{-14}$ is an effective coupling constant, and $C$ is some unknown constant.

While the above expression gives the cross section as a function of $\sqrt{\hat{s}}$ and the multiplicity of bosons, there are several issues. Firstly, this formula is only valid in a low energy regime, $\sqrt{\hat{s}} \ll M_{0}$, where $M_{0} \equiv \sqrt{6} \pi m_{W} \alpha_{W}^{-1} \sim 18 \mathrm{TeV}$. In fact, the cross section grows exponentially in this regime and eventually exceeds the $s$-wave unitarity bound ${ }^{9}$

$$
\hat{\sigma}_{\text {unitary }}^{s}(\sqrt{\hat{s}})=\frac{16 \pi}{\hat{s}},
$$

for $\sqrt{\hat{s}} \gtrsim M_{0}$. This motivates the phenomenological parametrisation of the sphaleron cross section:

$$
\hat{\sigma}(\sqrt{\hat{s}})=\min \left(\hat{\sigma}_{0}, \hat{\sigma}_{\text {unitary }}^{s}\right)
$$

with

$$
\hat{\sigma}_{0}(\sqrt{\hat{s}})=\frac{p_{\mathrm{sph}}}{m_{W}^{2}} \Theta\left(\sqrt{\hat{s}}-E_{\mathrm{thr}}\right),
$$

where $\Theta(x)$ is the Heaviside step function. The $(B+L)$-violating partonic cross section is parametrised by two unknown parameters, $p_{\mathrm{sph}}$ and $E_{\mathrm{thr}}$. The former is a dimensionless coefficient, which controls the overall size of the cross section. The latter represents the threshold energy where the partonic cross section is turned on, i.e. this is the scale at which the exponential suppression in the instanton formula is overcome by the exponential growth of cross section with the energy. Our cross section formula eq. (2.4) always respects the $s$-wave unitarity bound, as illustrated in figure 4 . This is in contrast to the previous phenomenological studies, e.g. [16, 17, 22, 44], where only an equation of the form of eq. (2.5) was used on its own to describe the partonic cross section.

\subsection{Parametrisation of boson distributions}

Our estimation of the boson multiplicity distributions is based on the LOME formula given by eq. (2.3). This formula predicts the total number of bosons to be $\left\langle n_{B}\right\rangle \gtrsim \frac{3}{2} \frac{\pi}{\alpha_{W}}\left(\frac{\sqrt{\hat{s}}}{M_{0}}\right)^{4 / 3}$ on average [45]. As was done in $[45,46]$, we fit the boson distributions generated from eq. (2.3) with the Gaussian: ${ }^{10}$

$$
\mathcal{P}\left(n_{B}, \hat{s} / \mu^{2}\right)=\exp \left(-\frac{\left(n_{B}-a\left(\hat{s} / \mu^{2}\right)^{2}\right.}{b\left(\hat{s} / \mu^{2}\right)}\right),
$$

where $n_{B}$ is the total boson multiplicity (i.e. $W, Z, \gamma$ and Higgs bosons) and $a$ and $b$ are functions of $\hat{s}$ in terms of some reference scale $\mu^{2}$. We note that for the fitted parameter values that were given in ref. [45], $b$ becomes negative at $\sqrt{\hat{s}} \simeq 57 \mathrm{TeV}$. Therefore, if those values are used, an absolute technical cut-off should be imposed at $\sqrt{\hat{s}} \simeq 50 \mathrm{TeV}$. However,

\footnotetext{
${ }^{9}$ Note that both the instanton and sphaleron field configurations are approximately spherically symmetric, hence the $s$-wave unitarity bound is expected to apply.

${ }^{10} \mathrm{We}$ note that the sign of the exponent in eq. (17) of [47] should be $(n-a)$ instead of $(n+a)$.
} 


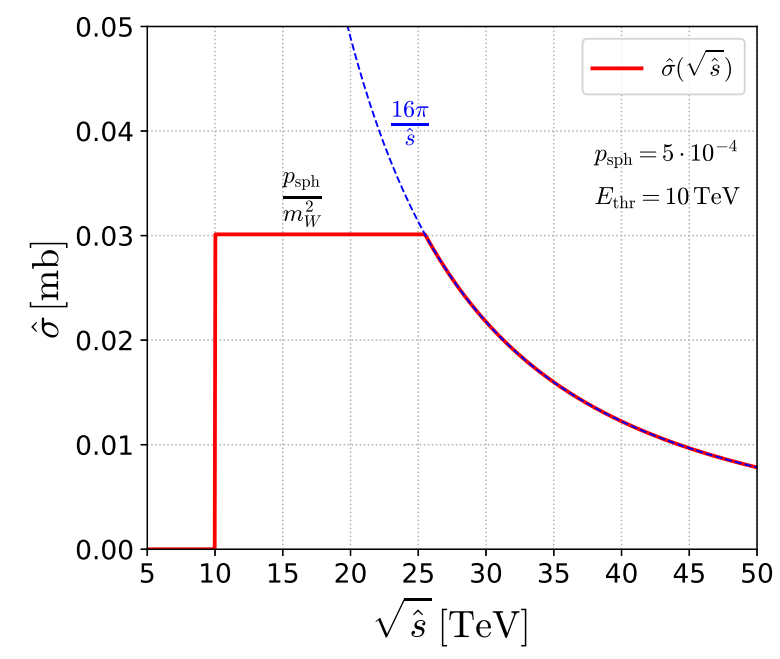

Figure 4. The partonic $(B+L)$-violating cross section, $\hat{\sigma}$, as a function of the centre of mass energy, $\sqrt{\hat{s}}$. The blue dashed line represents the $s$-wave unitarity bound.

we have performed an independent fit of the three curves at $\sqrt{\hat{s}}=16,17,18 \mathrm{TeV}$, appearing in figure 2 of ref. [46], obtaining the values:

$$
\begin{aligned}
& a=-19+6 \times 10^{-3} \sqrt{\hat{s} / \mathrm{GeV}^{2}}, \\
& b=7.4+3 \times 10^{-3} \sqrt{\hat{s} / \mathrm{GeV}^{2}}
\end{aligned}
$$

We use the above values in the phenomenological studies that involve a variable number of bosons. We note that sphaleron-induced final states are dominated by EW gauge boson production, and in all the studies of the present article we have set the number of Higgs bosons produced to zero [47].

As mentioned above, the LOME formula we rely on is valid only for $\sqrt{\hat{s}} \ll M_{0} \sim$ $18 \mathrm{TeV}$. Around and beyond this energy, no reliable theoretical estimation for the boson multiplicity is known. To obtain a phenomenological treatment of this uncertainty, we introduce an energy scale $E_{\text {freeze }}$ that acts as a transition point. That is, beyond $E_{\text {freeze }}>$ $M_{0}$, the $a$ and $b$ are fixed to their values at $\sqrt{\hat{s}}=E_{\text {freeze }}$. Since the boson multiplicity monotonically grows with $\sqrt{\hat{s}}$ in the LOME formula, this effectively introduces an upper bound to the total number of bosons that can be produced.

To see the effect of varying $E_{\text {freeze }}$, we show in figure 5 normalised distributions of the number of $W$ bosons (left) and all bosons (right) for $\sqrt{s}=27 \mathrm{TeV}, E_{\mathrm{thr}}=12 \mathrm{TeV}$ with three different values of $E_{\text {freeze }}: E_{\text {freeze }}=15 \mathrm{TeV}$ (red-solid), $20 \mathrm{TeV}$ (blue-dashed) and $25 \mathrm{TeV}$ (green-solid). One can see that the tail of the distributions become more suppressed as $E_{\text {freeze }}$ becomes smaller. This is because the LOME formula predicts larger multiplicities for larger $\sqrt{\hat{s}}$ but the boson multiplicity distribution is frozen for $\sqrt{\hat{s}}>E_{\text {freeze }}$ at $E_{\text {freeze }}$. This effect is more visible for $E_{\text {freeze }} \sim E_{\text {thr }}$ (red vs green histograms). For $E_{\text {freeze }} \gg E_{\text {thr }}$, the effect of $E_{\text {freeze }}$ is very small because the partonic collisions are dominated by $\sqrt{\hat{s}} \sim E_{\text {thr }}$ due to suppression of the parton density functions (PDF) (green vs blue histograms). ${ }^{11}$

\footnotetext{
${ }^{11}$ For the event generation we use the PDF4LHC15_nlo_mc PDF sets [48].
} 

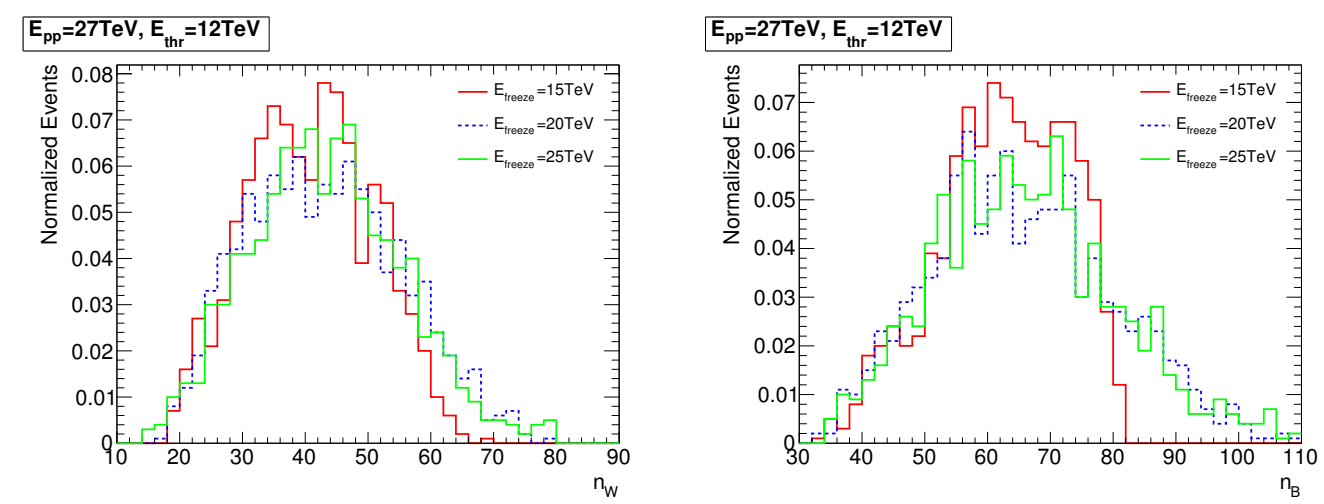

Figure 5. Normalised distributions of the number of $W$ bosons (left) and all bosons (right) for $\sqrt{s}=27 \mathrm{TeV}, E_{\mathrm{thr}}=12 \mathrm{TeV}$. The red-solid, blue-dashed and green-solid histograms correspond to $E_{\text {freeze }}=15,20 \mathrm{TeV}$ and $25 \mathrm{TeV}$, respectively.

\subsection{Multi-boson $B$ - and $L$-conserving processes}

To investigate the potential for observability of baryon- and lepton-number-violating processes at colliders, we must consider the relevant backgrounds that do not exhibit this property. Since sphaleron-induced processes are expected to contain a large number of bosons $n_{B} \gtrsim 1 / \alpha_{w} \sim 30$, they will subsequently decay to large numbers of jets - see the quantitative analysis of the next section. This implies that any Standard Model cross sections of perturbative background processes will be extremely small. However, it is expected that if the "signal" sphaleron-induced processes exist, there would also be accompanied with $B$ and $L$-conserving non-perturbative multi-boson processes with a similarly-large number of bosons [47]. The behaviour of such "multi-boson" processes is as uncertain as those of the sphaleron signal, but may occur at a similar rate.

We have also constructed a MC simulation of the multi-boson processes which behaves in an identical manner to that of sphaleron-induced processes in all aspects, apart from the quark "content" of the participating sub-processes. In this case, these are simply $2 \rightarrow 2$ scatterings, where we only allow $u$ and $d$ quarks in the initial state as in the sphaleron case. Since multi-boson processes are $B$ - and $L$-conserving, this implies that only the same $u$ and $d$ quarks appear in the final state. The number of colour configurations is accounted for as in the sphaleron case, i.e. by multiplying the event weight by an appropriate factor and then sampling uniformly at random. We do not investigate these processes as backgrounds in the phenomenological analysis that follows, but future studies should re-asses the results of ref. [47] which has considered discrimination between the $B$ - and $L$-violating and conserving processes. These processes are available in our MC event generator [49].

\section{Phenomenology at hadron colliders}

In this section we examine the experimental signatures of sphaleron-induced processes. In order to take into account the detector effects the generated hadronic event samples are passed to Delphes [50, 51]. Jets are reconstructed with the anti- $k_{T}$ algorithm [52] with distance parameter $R=0.4$ via the FastJet package [53]. Leptons ( $e^{ \pm}$and $\mu^{ \pm}$) 
and photons are required to be sufficiently isolated from other energetic particles around them. We calculate the scalar sum, $\mathcal{P}$, of the transverse momenta, $p_{T}$, of the neighbouring particles within $\Delta R=\sqrt{\Delta \eta^{2}+\Delta \phi^{2}}<r_{\max }$, and demand $\mathcal{P} / p_{T}^{i}<\mathcal{I}_{\max }$ for isolation, where $p_{T}^{i}$ is the magnitude of transverse momentum of the lepton or photon to be isolated. We take $r_{\max }=0.3$ for electrons and photons and 0.4 for muons. $\mathcal{I}_{\max }$ is taken to be 0.10 , 0.12 and 0.15 for electrons, photons and muons, respectively. Finally, we require all objects (jets, leptons and photons) to have $p_{T}>30 \mathrm{GeV}$ and pseudo-rapidity $|\eta|<5$ (jets), $<2.4$ (muons) and $<2.5$ (electrons and photons).

\subsection{Reconstructed distributions}

In this subsection we study the impact of some parameters that are present in our phenomenological model, such as $\sqrt{s}$ and $E_{\mathrm{thr}}$, on several multiplicity and kinematic distributions. We start by showing in figure 6 , the normalised distributions of the inclusive multiplicity (left) and the transverse momentum of the hardest jet, $p_{T}^{j 1}$, in the events (right) for collider energies $\sqrt{s}=13 \mathrm{TeV}$ (top), $27 \mathrm{TeV}$ (middle) and $100 \mathrm{TeV}$ (bottom). We take $E_{\text {freeze }}=15 \mathrm{TeV}$ for $\sqrt{s}=13 \mathrm{TeV}$ and $E_{\text {freeze }}=20 \mathrm{TeV}$ for $27,100 \mathrm{TeV}$, but the dependence on $E_{\text {freeze }}$ of these distributions was found to be rather mild. For $\sqrt{s}=13 \mathrm{TeV}$ we show four histograms corresponding to $E_{\text {thr }}=8 \mathrm{TeV}$ (red-solid), $9 \mathrm{TeV}$ (pink-dashed), $10 \mathrm{TeV}$ (blue-solid), $11 \mathrm{TeV}$ (cyan-dashed), while for the $27 \mathrm{TeV}$ and $100 \mathrm{TeV}$ colliders, we examined five values of $E_{\mathrm{thr}}: 9 \mathrm{TeV}$ (red), $12 \mathrm{TeV}$ (pink-dashed), $14 \mathrm{TeV}$ (blue), $16 \mathrm{TeV}$ (cyan-dashed) and $18 \mathrm{TeV}$ (green-solid).

The inclusive multiplicity, denoted as $N\left(p_{T}>100\right)$, is defined as the number of reconstructed objects (jets, leptons and photons satisfying the isolation criteria) with $p_{T}>100 \mathrm{GeV}$. These high- $p_{T}$ objects may originate either from direct anti-quark plus anti-lepton production in the hard interaction through the operator in eq. (2.1), or from secondary decays of the produced massive EW gauge bosons. For $\sqrt{s}=13 \mathrm{TeV}$ (top-left) we see that the $N\left(p_{T}>100\right)$ distribution peaks at around $15-17$ objects and the multiplicity is slightly larger for larger $E_{\mathrm{thr}}$ within the variation taken here: $E_{\mathrm{thr}}=(9-11) \mathrm{TeV}$. For $\sqrt{s}=27 \mathrm{TeV}$ and $100 \mathrm{TeV}$ (middle-left and bottom-left, respectively), the multiplicity distributions are shifted to larger values compared to those at the $13 \mathrm{TeV}$ LHC. For both the $27 \mathrm{TeV}$ and $100 \mathrm{TeV}$ colliders, it peaks at $N\left(p_{T}>100\right) \sim 17$ for $E_{\mathrm{thr}}=9 \mathrm{TeV}$ and at $N\left(p_{T}>100\right) \sim 22$ for $E_{\mathrm{thr}}=18 \mathrm{TeV}$. We also observe that the distributions are broader for $\sqrt{s}=100 \mathrm{TeV}$ than for $27 \mathrm{TeV}$.

The right-hand-side plots of figure 6 show the transverse momentum of the hardest jet, $p_{T}^{j 1}$. We expect that the hardest jet is likely to originate from one of the anti-quarks produced in the primary interaction, through the operator in eq. (2.1). At the $13 \mathrm{TeV}$ LHC (top-right), the distribution peaks around $p_{T}^{j 1} \sim 700 \mathrm{GeV}$ and the dependence of $E_{\mathrm{thr}}$ is rather modest in the range we examined, $E_{\mathrm{thr}} \in(8-11) \mathrm{TeV}$. Additionally, these distributions are somewhat narrow and confined below $p_{T}^{j 1} \sim 1.5 \mathrm{TeV}$. Contrary to them, the distributions for the $27 \mathrm{TeV}$ (middle-right) and $100 \mathrm{TeV}$ (bottom-right) colliders are more dependent on $E_{\mathrm{thr}}$ in the range we considered, $E_{\mathrm{thr}} \in(9-18) \mathrm{TeV}$. The peak position varies from $p_{T}^{j 1} \sim 800 \mathrm{GeV}$ to $p_{T}^{j 1} \sim 1.5 \mathrm{TeV}$ when $E_{\text {thr }}$ is changed from 9 to $18 \mathrm{TeV}$. We also note that the $p_{T}^{j 1}$ distributions become broader for larger $E_{\mathrm{thr}}$, as well as for larger 

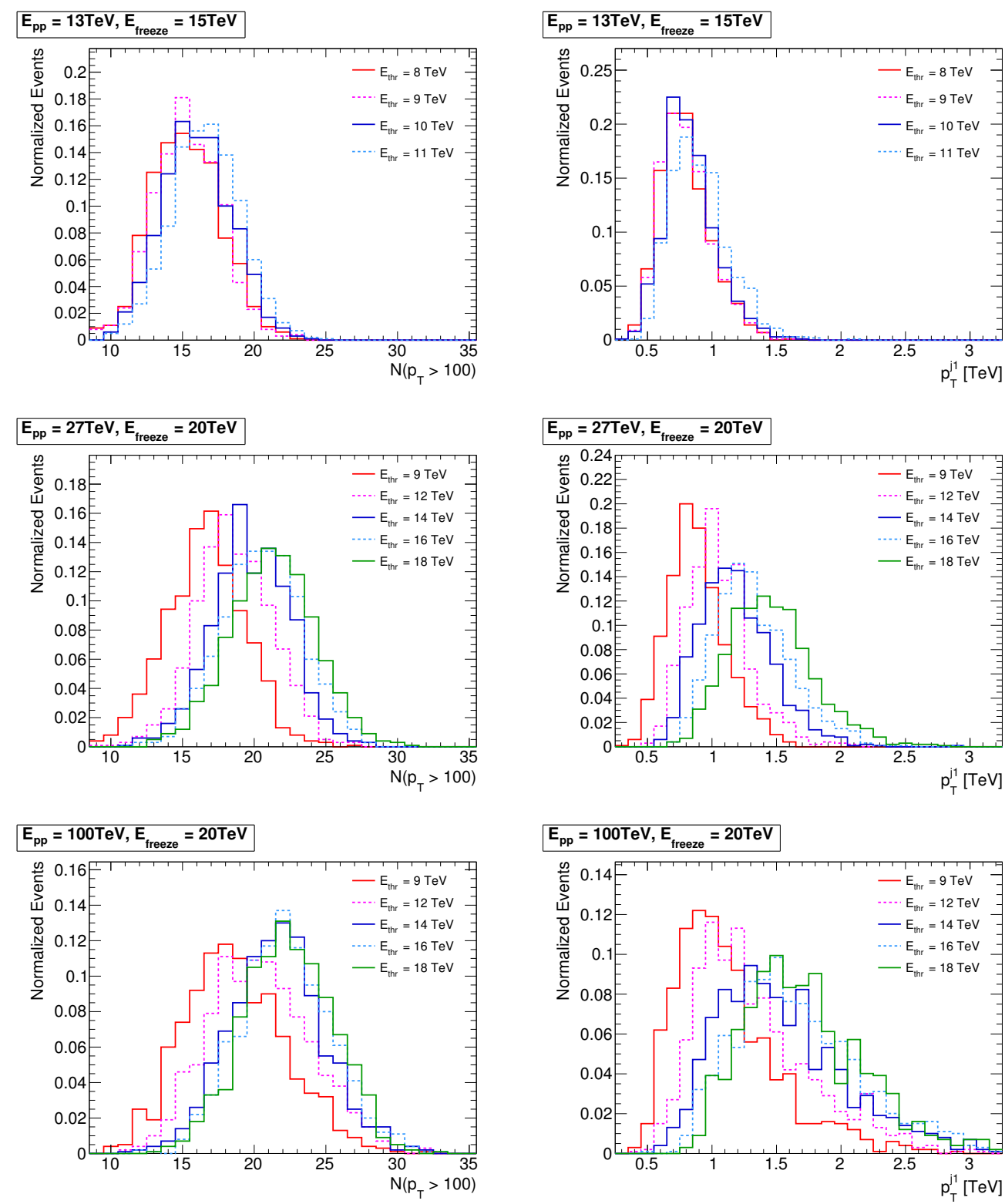

Figure 6. Distributions of the inclusive reconstructed object multiplicity $N\left(p_{T}>100\right)$ (left) and the $p_{T}$ of the hardest jet in the events (right) for $\sqrt{s}=13 \mathrm{TeV}$ (top), $27 \mathrm{TeV}$ (middle) and $100 \mathrm{TeV}$ (bottom). We set $E_{\text {freeze }}=15 \mathrm{TeV}$ for $\sqrt{s}=13 \mathrm{TeV}$, whereas for $\sqrt{s}=27$ and $100 \mathrm{TeV}$ we adopt $E_{\text {freeze }}=20 \mathrm{TeV}$.

collider energies. This is due to the fact that the phase-space volume is bigger for larger $E_{\text {thr }}$ and therefore it is easier for the hardest jet to attain a higher transverse momentum. At higher collider energies, the distributions become broader because the PDF suppression becomes milder at higher partonic collision energies.

So far we have generated the boson multiplicity according to the LOME formula. To see the effect of the boson multiplicity on the jet transverse momentum and the number of high- $p_{T}$ objects, we look at these distributions with a fixed boson multiplicity, $n_{B}$. In 

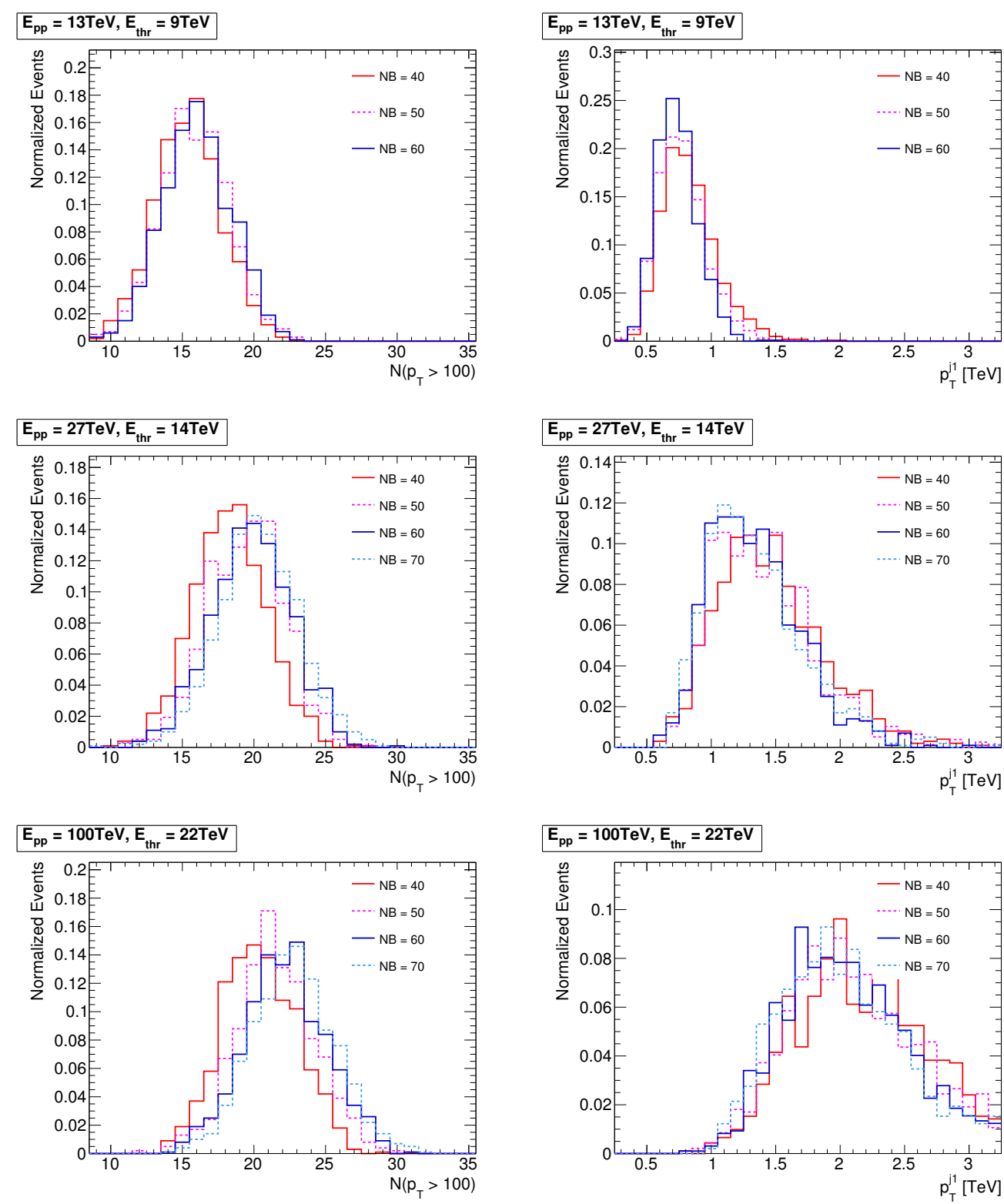

Figure 7. The same as figure 6 but with the number of total gauge bosons fixed to $n_{B}=40$ (red-solid), 50 (pink-dashed) and 60 (blue-solid) for the $13 \mathrm{TeV}$ LHC. In addition to these, the case $n_{B}=70$ (cyan-dashed) is shown for $\sqrt{s}=27$ and $100 \mathrm{TeV}$. The threshold energy is taken as $E_{\mathrm{thr}}=9,14$ and $22 \mathrm{TeV}$ for $\sqrt{s}=13,27$ and $100 \mathrm{TeV}$.

figure 7, we show the distributions of $N\left(p_{T}>100\right)$ (left) and of the hardest jet $p_{T}$ for a fixed number of total EW gauge bosons. For the $13 \mathrm{TeV}$ LHC (top panels), we show histograms for $n_{B}=40$ (red-solid), 50 (pink-dashed) and 60 (blue-solid). For $27 \mathrm{TeV}$ (middle) and $100 \mathrm{TeV}$ (bottom) colliders, in addition to those, the case $n_{B}=70$ (cyan-dashed) is shown. We take $E_{\mathrm{thr}}=9,14 \mathrm{TeV}$ and $22 \mathrm{TeV}$ for $\sqrt{s}=13,27$ and $100 \mathrm{TeV}$, respectively.

In the right-hand plots of figure 7 we see that the number of high- $p_{T}$ reconstructed objects with $p_{T}>100 \mathrm{GeV}$ has a mild dependence on $n_{B}$, though one can observe that 
$N\left(p_{T}>100\right)$ increases with $n_{B}$. This is expected because the EW gauge bosons decay into jets and leptons and as long as they are isolated and have $p_{T}>100 \mathrm{GeV}$, they contribute to $N\left(p_{T}>100\right)$. On the other hand, $N\left(p_{T}>100\right)$ rather strongly depends on the collider energy and $E_{\mathrm{thr}}$. The peak of the $N\left(p_{T}>100\right)$ distribution sits around 16, 20 and 22 objects for $\left(\sqrt{s}, E_{\mathrm{thr}}\right)=(13,9),(27,14),(100,22) \mathrm{TeV}$, respectively. We also note that the distributions are broader for larger $\sqrt{s}$ and $E_{\mathrm{thr}}$, as we have seen in figure 6 .

The right-hand-side plots figure 7 show the distribution of the hardest jet transverse momentum, $p_{T}^{j 1}$. Just as for $N\left(p_{T}>100\right)$, its dependence on $n_{B}$ is mild. There is a tendency for the $p_{T}^{j 1}$ to become softer for larger $n_{B}$. This is because for a fixed partonic energy, larger $n_{B}$ implies a smaller amount of energy available to be shared among the primary particles (i.e. anti-fermions, EW gauge bosons). As was the case for $N\left(p_{T}>100\right)$, the distributions are broader and harder at larger collider energies and $E_{\mathrm{thr}}$.

In figure 8 , we show the distributions of the number of isolated leptons, $N_{\text {lep }}$, (left) and photons, $N_{\gamma}$, (right) with $p_{T}>30 \mathrm{GeV}$ for $\sqrt{s}=13 \mathrm{TeV}$ (top), $27 \mathrm{TeV}$ (middle) and $100 \mathrm{TeV}$ (bottom). The choice of $E_{\text {freeze }}$ and $E_{\text {thr }}$ and the line-style discriminating different $E_{\text {thr }}$ are the same as in figure 6 . In sphaleron events, high- $p_{T}$ leptons are produced either primarily from the hard interaction via the operator in eq. (2.1) or secondarily from the decay of massive EW bosons. ${ }^{12}$ In the top-right plot, we see that the sphaleron events contain at least one isolated lepton with $p_{T}>30 \mathrm{GeV}$ more than half of the time. Unlike the inclusive multiplicity $N\left(p_{T}>100\right), N_{\text {lep }}$ depends very weakly on the threshold energy $E_{\text {thr }}$. Comparing these distributions to those with higher collider energies (middle-left and bottom-left plots), we can observe that the number of isolated leptons decreases as the $\sqrt{s}$ increases. This is, at first glance, counter-intuitive because, as we have seen above, events at a higher energy collider contain more massive EW gauge bosons, and one may expect there will be more leptons coming from decays of those. However, events with a very large number of massive EW bosons typically also end up containing a large number of jets coming from their decays. Consequently, in such a busy environment the lepton isolation criteria are more likely to fail, suppressing the total number of isolated leptons. In the middle-left plot, with $\sqrt{s}=27 \mathrm{TeV}$, we also observe that $N_{\text {lep }}$ is larger for $E_{\text {thr }}=9 \mathrm{TeV}$ than for $E_{\mathrm{thr}}=14 \mathrm{TeV}$. This can also be understood by the same reasoning: the multiplicity of EW gauge bosons is larger in general for higher $E_{\mathrm{thr}}=14$ and $16 \mathrm{TeV}$. One can also notice that the lepton multiplicity is slightly larger for $E_{\mathrm{thr}}=18 \mathrm{TeV}$ than for $E_{\mathrm{thr}}=14$ and $16 \mathrm{TeV}$ in the middle-left and bottom-left plots. This is because the number of EW bosons does not increase much from $E_{\text {thr }}=16$ to $18 \mathrm{TeV}$ since the multiplicity freezes out at $\sqrt{\hat{s}}=E_{\text {freeze }}=20 \mathrm{TeV}$. This implies that the leptons are more likely to be accepted for $E_{\mathrm{thr}}=18 \mathrm{TeV}$ because they are more energetic on average and are more likely to satisfy the $p_{T}$ cut.

We now turn our attention to the right-hand-side plots of figure 8 , in order to discuss the multiplicity of the isolated photons. In sphaleron events, photons can be produced from the primary interaction. Roughly speaking, a neutral $S U(2)$ gauge boson is converted to a photon or a $Z$-boson with probabilities $\sin ^{2} \theta_{W}$ and $\cos ^{2} \theta_{W}$, respectively. Photons may

\footnotetext{
${ }^{12}$ They can also be produced in the decays of tau-leptons and heavy hadrons.
} 

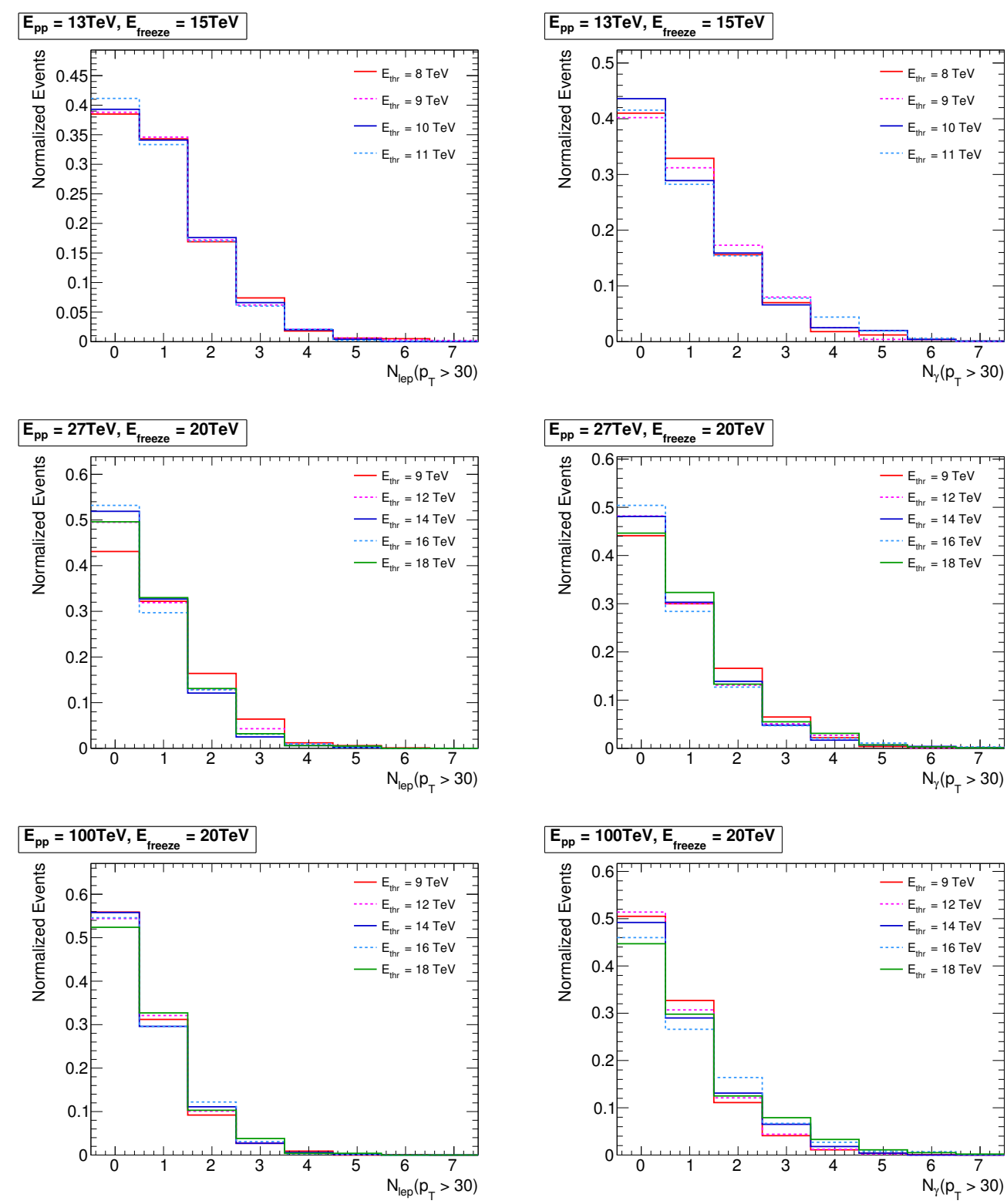

Figure 8. Multiplicity distributions of isolated leptons (left) and photons (right) for $\sqrt{s}=13 \mathrm{TeV}$ (top), $27 \mathrm{TeV}$ (middle) and $100 \mathrm{TeV}$ (bottom). The choice of values for $E_{\text {freeze }}$ and $E_{\mathrm{thr}}$, as well as the line-styles and colour schemes are as in figure 6 .

also originate from initial and final state electromagnetic radiation and hadronic decays. As can be seen, the multiplicity distribution of isolated photons is, by coincidence, similar to that of isolated leptons. In general an event contains at least one photon about a half of the time. We see the same tendency as in the case of the leptons: the multiplicity becomes smaller at larger collider energies. This stems from the same fact as for the case of the leptons; i.e. that the isolation criteria are likely to fail due to a busy environment.

In figure 9 we show the same $N_{\text {lep }}$ and $N_{\gamma}$ distributions at fixed $n_{B}$. The same linestyles and colour schemes are used as in figure 7 to discriminate the different $n_{B}$. In 

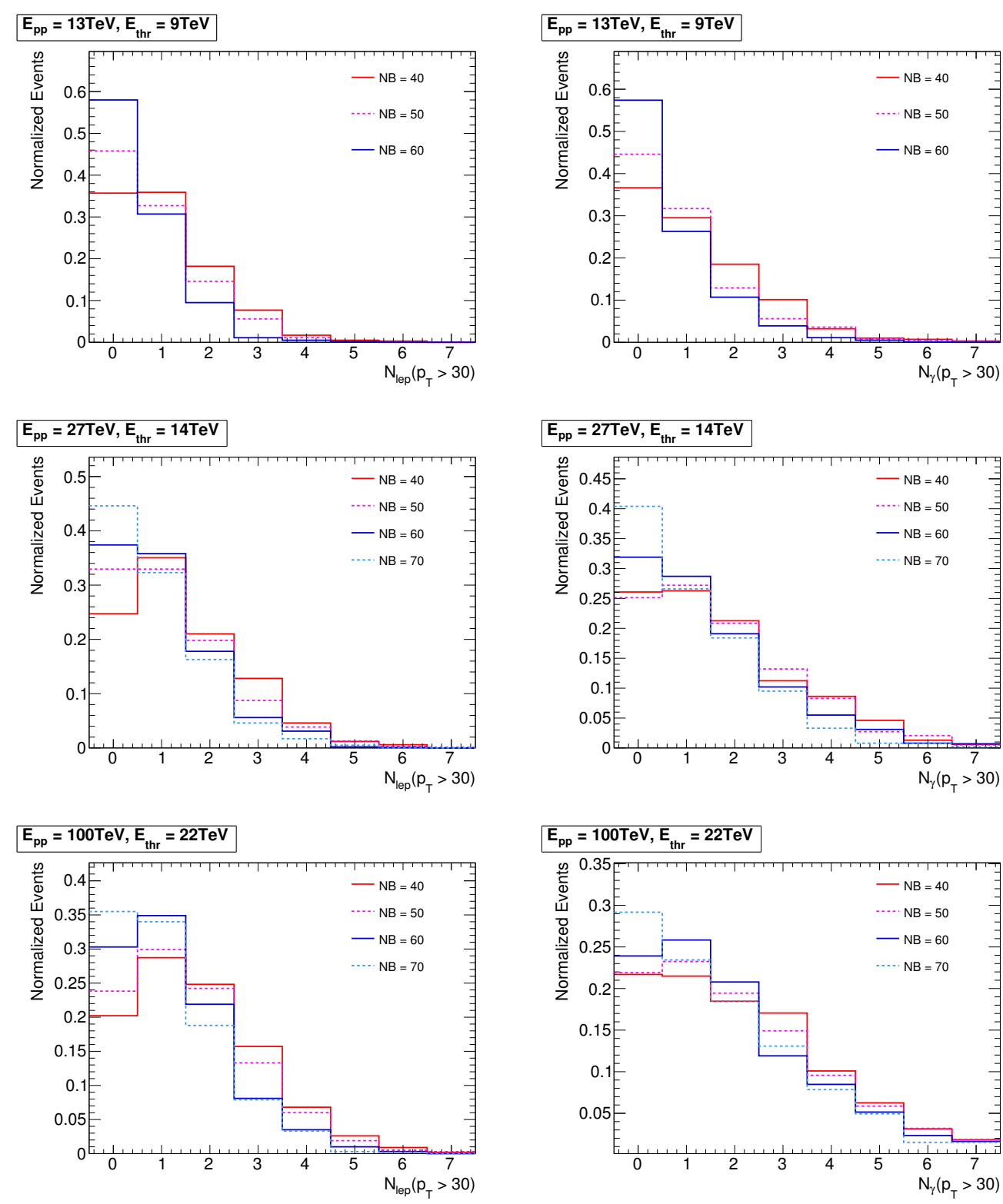

Figure 9. The same as figure 8 but with the total numbers of bosons instead fixed to $n_{B}=40$ (red-solid), 50 (pink-dashed) and 60 (blue-solid) for the $13 \mathrm{TeV}$ LHC. In addition to these $n_{B}=70$ (cyan-dashed) is shown for $\sqrt{s}=27$ and $100 \mathrm{TeV}$. The threshold energy is taken as $E_{\mathrm{thr}}=9,14 \mathrm{TeV}$ and $22 \mathrm{TeV}$ for $\sqrt{s}=13,27 \mathrm{TeV}$ and $100 \mathrm{TeV}$.

these plots we can consistently see that the lepton and photon multiplicities are larger in general for smaller $n_{B}$. This is in agreement with our expectations of the EW gauge boson multiplicity and the isolation criteria. Unlike the case in figure 8 , the multiplicity becomes larger at larger collider energies. This is because, for a fixed $n_{B}$, the effect of increasing $\sqrt{s}$ is to make the leptons and photons more energetic on average, which helps in satisfying the $p_{T}$ cut. We note that for the $100 \mathrm{TeV}$ collider with a lower boson multiplicity $\left(n_{B} \sim 40\right)$, the events tend to have many photons. There will be $N_{\gamma} \geq 2$ more than half of the time 
in this particular case $\left(\sqrt{s}=100 \mathrm{TeV}, n_{B}=40\right)$. Exploiting this fact may help to reduce the SM background in future searches.

\subsection{Projected sensitivities}

In the present subsection we estimate the sensitivities of observing sphaleron-induced processes at proton-proton colliders. We consider three cases; the $13 \mathrm{TeV}$ high-luminosity LHC (HL-LHC13), the $27 \mathrm{TeV}$ high-energy LHC (HE-LHC27) and a future $100 \mathrm{TeV}$ circular collider (FCC100). To facilitate the comparison of the capability of each collider, we assume the envisioned integrated luminosity of the HL-LHC13, $3 \mathrm{ab}^{-1}$, in all three cases, although future collider experiments will likely collect more data.

Our analysis is inspired by the CMS analysis of ref. [44], on searches for sphaleron and mini black hole production in $13 \mathrm{TeV}$ data with $L=36 \mathrm{fb}^{-1}$. Following this analysis, we apply cuts on two variables; $N\left(p_{T}>100\right)$ and $S_{T}^{100}$. The former variable is defined as before, as the number of reconstructed objects (jets, isolated leptons and photons) with $p_{T}>100 \mathrm{GeV}$ and the latter variable is the scalar sum of the transverse missing energy $\left(\mathbb{E}_{T}\right)$ and $p_{T}$ of all reconstructed objects with $p_{T}>100 \mathrm{GeV}$. Our signal selection requirement is given by

$$
\begin{aligned}
& N\left(p_{T}>100\right) \geq 11, S_{T}^{100}>4 \mathrm{TeV} \cdots \mathrm{HL}-\mathrm{LHC} 13 \\
& N\left(p_{T}>100\right) \geq 15, S_{T}^{100}>7 \mathrm{TeV} \cdots \mathrm{HE}-\mathrm{LHC} 27, \mathrm{FCC} 100
\end{aligned}
$$

It has been reported in the CMS analysis [44] that the SM background is reduced to less than 1 event, with the above HL-LHC13 selection criteria, while the signal efficiency remains greater than $90 \%$ [22]. For the HE-LHC27 and FCC100, we have increased these requirements upwards. We expect that the SM background for these cases is also reduced to less than 1 event.

The impact of the $N\left(p_{T}>100\right)$ cut on the signal efficiency can be deduced from examining the left panel of figure 6 and 7 . One can see that signal events are localised above $N\left(p_{T}>100\right) \gtrsim 11$ for $\sqrt{s}=13 \mathrm{TeV}$ and $\gtrsim 15$ for $\sqrt{s}=27$ and $100 \mathrm{TeV}$. Therefore, the signal selection efficiency is almost $100 \%$ for this cut.

To see the efficiency for the $S_{T}^{100}$ selection, we show the distributions of $S_{T}^{100}$ in figure 10. In the left-hand side plots, multiplicity distributions are constructed based on the LOME prescription, while the boson multiplicity is fixed in the right-hand-side plots. Before plotting, events are required to satisfy $N\left(p_{T}>100\right) \geq 11$ for $\sqrt{s}=13 \mathrm{TeV}$ and $N\left(p_{T}>\right.$ $100) \geq 15$ for $\sqrt{s}=27$ and $100 \mathrm{TeV}$ so that the area of the histogram with $S_{T}^{100}>4(7)$ $\mathrm{TeV}$ corresponds the signal efficiency of our event selection. In the top two plots, we see that the signal efficiency for the HL-LHC13 selection is $\gtrsim 90 \%$. In the middle-left and bottom-right plots, one can see that the $S_{T}^{100}$ distribution is sensitive to $E_{\text {thr. }}$. For $\sqrt{s}=27$ and $100 \mathrm{TeV}$, the efficiency can be larger than $80 \%$ for $E_{\mathrm{thr}} \gtrsim 12 \mathrm{TeV}$. The signal efficiency is still as large as $50 \%$ even for $E_{\mathrm{thr}} \sim 9 \mathrm{TeV}$.

In the plots on the right-hand side, we see that the $S_{T}^{100}$ distribution is not sensitive to $n_{B}$. This is expected because the variable is defined as the sum of all transverse momenta and, e.g. splitting one particle into two does not change the sum of the $p_{T}$, as long as all particles are counted correctly. 



Figure 10. The $S_{T}$ distributions for $\sqrt{s}=13 \mathrm{TeV}$ (top), $27 \mathrm{TeV}$ (middle) and $100 \mathrm{TeV}$ (bottom). On the left-hand-side plots, multiplicity distributions have been generated based on the LOME formula, while the boson multiplicity was fixed on the right-hand-side panels. The same line-styles and colour scheme are used to discriminate between the different $E_{\mathrm{thr}}$ and $n_{B}$ as in figure 6 and figure 7 . The $N_{100} \geq 11$ (15) cut is applied for $\sqrt{s}=13(27$ and 100$) \mathrm{TeV}$.

Our goal is to derive the projected limit on the $E_{\text {thr }}$ vs $p_{\text {sph }}$ plane. For this purpose, we first calculate the cross section of sphaleron-induced processes in terms of $E_{\text {thr }}$ setting $p_{\mathrm{sph}}=1$. We convolve the partonic cross section defined by eqs. (2.4) and (2.5) with the PDFs. In particular, the leading order CT10 PDF set is used here [54]. The resulting hadronic cross sections for $p_{\mathrm{sph}}=1$ are shown in figure 11 . We see that the hadronic cross sections are quite large for smaller $E_{\mathrm{sph}}$. At $E_{\mathrm{sph}}=9 \mathrm{TeV}$, we find $\sigma \simeq 10,10^{5}$ and $10^{7} \mathrm{fb}$ 

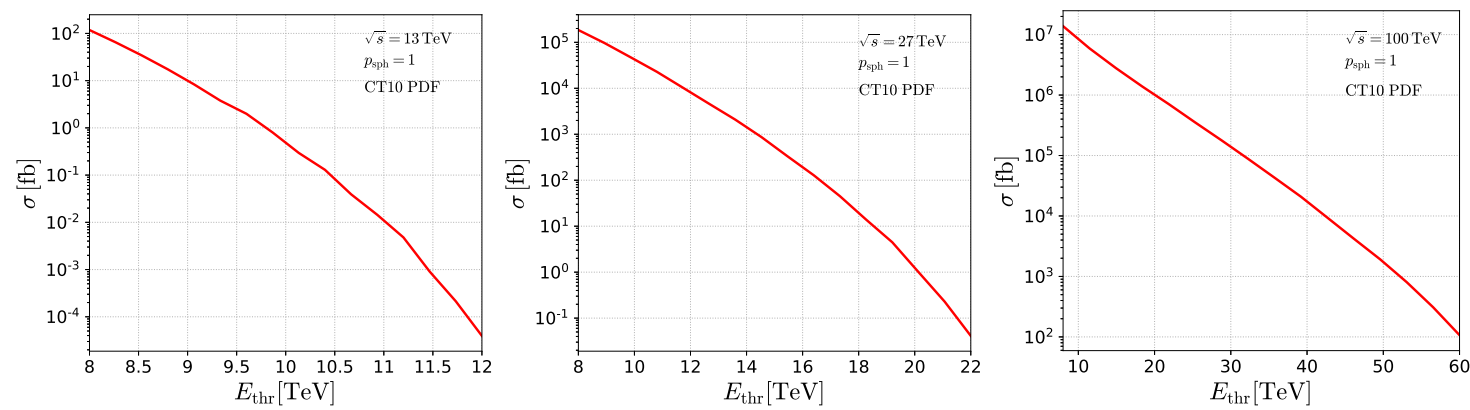

Figure 11. The cross section for the sphaleron-induced processes as a function of $E_{\mathrm{thr}}$ with $p_{\mathrm{sph}}=1$. The proton-proton collision energy is taken to be $13 \mathrm{TeV}$ (left), $27 \mathrm{TeV}$ (middle) and $100 \mathrm{TeV}$ (right). The leading order CT10 PDF set was used.

for $\sqrt{s}=13,27$ and $100 \mathrm{TeV}$, respectively. The cross section falls rapidly as $E_{\text {thr }}$ increases, but this reduction is faster (slower) for smaller (larger) collider energies. For example, for $\sqrt{s}=13 \mathrm{TeV}$, the cross section is reduced to $10^{-(4-5)} \mathrm{fb}$ at $E_{\mathrm{thr}}=12 \mathrm{TeV}$. On the other hand, for $\sqrt{s}=100 \mathrm{TeV}$, the cross section remains as large as $10^{5} \mathrm{fb}$ even for $E_{\mathrm{thr}}=30 \mathrm{TeV}$.

With these considerations at hand, we can derive the projected sensitivities. We estimate the signal yield by

$$
N_{s}=\sigma \cdot \epsilon \cdot L_{\text {int }},
$$

where $\sigma$ is hadronic cross section, $\epsilon$ is the signal efficiency and $L_{\text {int }}$ is the integrated luminosity.

Since precise estimation of the multi-particle SM background is theoretically challenging and depends substantially on detector performance, we follow here a simplified approach. We assume that the SM background is well-suppressed below $\mathcal{O}(1)$ due to the selection cuts, and we highlight the region between $N_{s}=3$ and 10 in the $E_{\text {thr }}$ vs $p_{\text {sph }}$ plane. We expect that the boundary of this region will roughly correspond to the exclusion and discovery of the sphaleron-induced processes.

Figure 12 shows the sensitivity of the HL-LHC13 (top), HE-LHC27 (middle) and FCC100 (bottom) to the sphaleron-induced processes in the $E_{\text {thr }}$ vs $p_{\text {sph }}$ plane. On the left-hand side, the boson multiplicity has been generated according to the LOME formula, while on the right-hand side it has been fixed to either $n_{B}=40$ or 70 . The light red bands in the left-hand-side plots denote the region where the collider is sensitive to the sphaleron processes, $N_{s} \in(3-10)$. On the right-hand side we show only the boundary of this region, $N_{s}=3,10$, and distinguish different $n_{B}$ with different lines.

First of all, it is clear that the impact of boson multiplicity on the sensitivity is very mild. This is evident by noticing the similarities between left-hand- and right-hand-side plots, as well as between different lines in the right-hand-side plots. There exists only a mild dependence on $n_{B}$ in the region around $E_{\text {thr }} \sim 9 \mathrm{TeV}$ for the HE-LHC27 (middle-right plot). This is because the efficiency of the $N\left(p_{T}>100\right) \geq 15$ cut differs slightly between $n_{B}=40$ and 70 , as can be seen in the middle-left plot in figure 6 . 

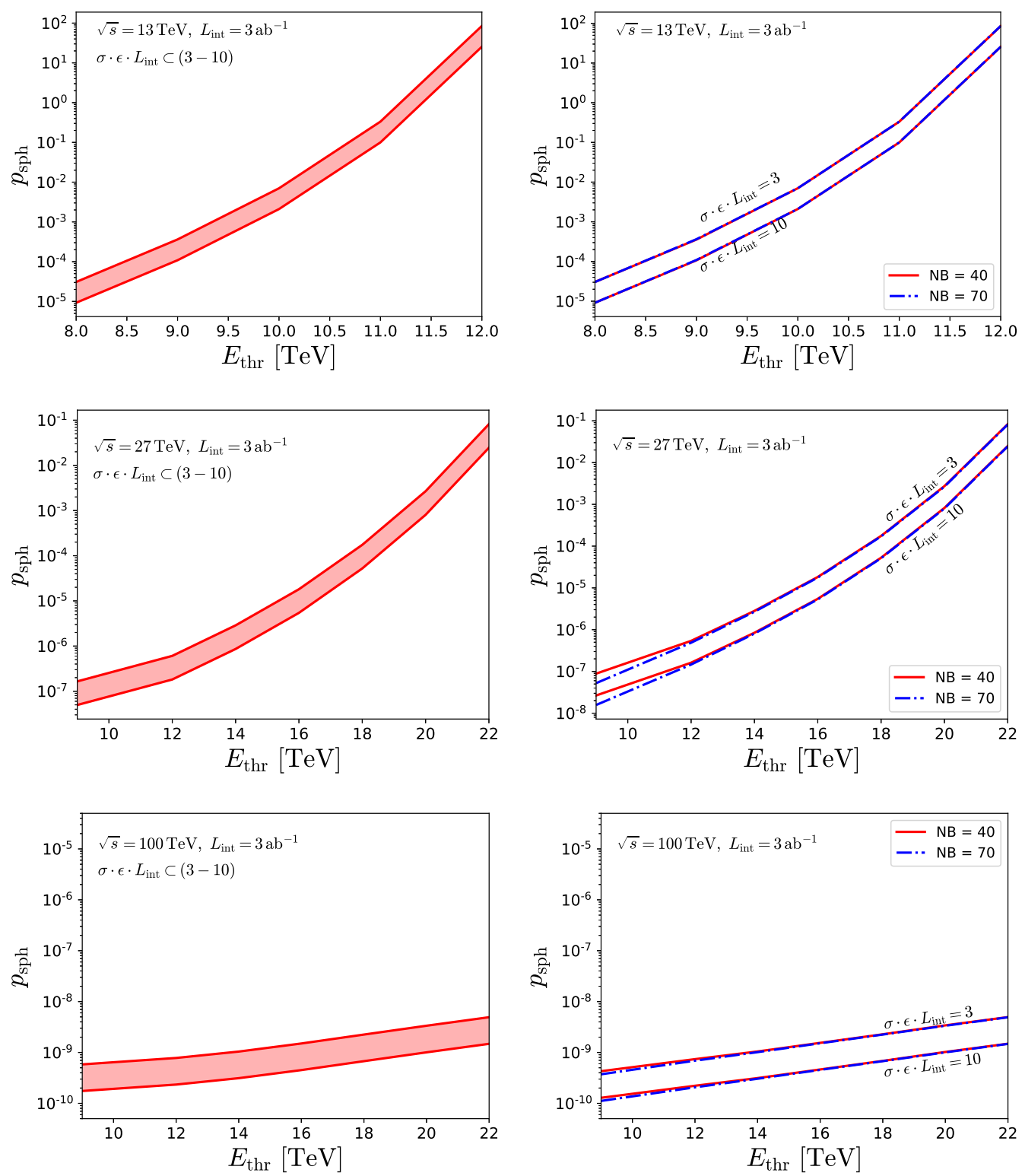

Figure 12. The projected sensitivities in the $E_{\text {thr }}$ vs $p_{\text {sph }}$ plane at the HL-LHC13 (top), HE-LHC27 (middle) and FCC100 (bottom). In the right panel, we generate boson multiplicity according to the LOME formula, while we fix the boson multiplicity to $n_{B}=40$ and 70 . On the left-hand side, $E_{\text {freeze }}$ is taken to be $15(20) \mathrm{TeV}$ for $\sqrt{s}=13(27,100) \mathrm{TeV}$.

In the top two plots, one can see that HL-LHC13 can probe values of $p_{\mathrm{sph}}$ up to $\sim 10^{-4}$ for $E_{\text {thr }} \sim 9 \mathrm{TeV}$. It is interesting to note that recent studies estimated the threshold energy to lie around this scale $[14,15]$. The high-luminosity LHC will therefore provide a meaningful constraint on this type of estimation. In the middle two plots, we see that the reach of HE-LHC27 (middle plots) performs much better than HL-LHC13. In particular, one can exclude $p_{\text {sph }} \gtrsim 10^{-3}$ at $E_{\text {thr }} \lesssim 20 \mathrm{TeV}$. In fact, some studies have suggested that $E_{\text {thr }}$ may be around $20 \mathrm{TeV}[12,13]$, and therefore the HE-LHC27 will be capable of 
providing important information on the rate of sphaleron processes up to this limit. Finally we turn our attention to the bottom plots, showing the results for the FCC100. The reach of this collider is much higher, probing e.g. values of the parameter $p_{\text {sph }} \gtrsim 10^{-9}$ even up to threshold scales of $E_{\mathrm{thr}} \sim 22 \mathrm{TeV}$. Therefore, a $100 \mathrm{TeV}$ collider would provide a strong constraint to the rate of EW sphaleron processes in the SM.

\section{Conclusions}

We have developed a modern event generator that models sphaleron-induced processes within the Herwig general-purpose Monte Carlo framework. ${ }^{13}$ This event generator captures the gross theoretical features of these baryon- and lepton-number-violating processes, inspired by theoretical considerations of the fermionic (flavour) and bosonic content of the final states. We have employed this development to perform phenomenological studies at hadron colliders such as the LHC $(13 \mathrm{TeV})$, as well as a high-energy upgrade $(27 \mathrm{TeV})$ and at the Future Circular Collider $(100 \mathrm{TeV})$. We have examined various relevant reconstructed distributions, describing in detail their behaviour with respect to variations of the model parameters. Our analysis demonstrates that for a wide range of the parameters of the model considered, all three colliders will provide meaningful constraints on the rates of baryon- and lepton-number violating processes at various energy scales with $3 \mathrm{ab}^{-1}$ of integrated luminosity. In particular, through the $p_{\text {sph }}$ parameter, appearing in the parametrisation of the cross sections of eqs. (2.4) and (2.5), the HL-LHC at $13 \mathrm{TeV}$ can constrain $p_{\text {sph }}$ up to $\sim 10^{-4}$ for a threshold scale of $9 \mathrm{TeV}$, the HE-LHC at $27 \mathrm{TeV}$ could provide $p_{\mathrm{sph}} \gtrsim 10^{-3}$ at $E_{\mathrm{thr}} \lesssim 20 \mathrm{TeV}$ and the FCC-hh at $100 \mathrm{TeV}$ could probe values of this parameter $p_{\mathrm{sph}} \gtrsim 10^{-9}$ up to threshold scales of $E_{\mathrm{thr}} \sim 22 \mathrm{TeV}$. Given these limits, our study motivates the inclusion of the study of sphaleron-induced processes in current or future collider programmes; it is quite likely that such an endeavour will illuminate the observed matter-anti-matter asymmetry of the Universe.

\section{Acknowledgments}

We would like to thank Bryan Webber for stimulating discussions in the duration of this project. AP is also grateful for interesting discussions with Bert Schellekens, Marieke Postma, Chris Korthals-Altes and Jan Smit and acknowledges support by the ERC grant ERC-STG-2015-677323. This work is supported by the Netherlands National Organisation for Scientific Research (NWO) that is funded by the Dutch Ministry of Education, Culture and Science (OCW). In particular, AP is supported by the NWO D-ITP consortium. The work of KS is partially supported by the National Science Centre, Poland, under research grants 2017/26/E/ST2/00135 and the Beethoven grants DEC-2016/23/G/ST2/04301. SP is grateful for the kind hospitality of Mainz Institute for Theoretical Physics (MITP) of the DFG Cluster of Excellence PRISMA ${ }^{+}$(project ID 39083149), where this work has been finalised. This work has been supported in part by the European Union's Horizon 2020 research and innovation programme as part of the Marie Skłodowska-Curie Innovative

\footnotetext{
${ }^{13}$ This event generator acts as a plug-in to Herwig and can be retrieved from the repository [49].
} 
Training Network MCnetITN3 (grant agreement no. 722104). SP acknowledges partial support by the COST actions CA16201 "PARTICLEFACE" and CA16108 "VBSCAN".

Open Access. This article is distributed under the terms of the Creative Commons Attribution License (CC-BY 4.0), which permits any use, distribution and reproduction in any medium, provided the original author(s) and source are credited.

\section{References}

[1] G. 't Hooft, Symmetry breaking through Bell-Jackiw anomalies, Phys. Rev. Lett. 37 (1976) 8 [INSPIRE].

[2] Wikipedia, Per aspera ad astra - Wikipedia, the free encyclopedia (2019).

[3] A. Ringwald, High-energy breakdown of perturbation theory in the electroweak instanton sector, Nucl. Phys. B 330 (1990) 1 [InSPIRE].

[4] O. Espinosa, High-energy behavior of baryon and lepton number violating scattering amplitudes and breakdown of unitarity in the standard model, Nucl. Phys. B 343 (1990) 310 [INSPIRE].

[5] P.B. Arnold and L.D. McLerran, The sphaleron strikes back, Phys. Rev. D 37 (1988) 1020 [INSPIRE].

[6] L.D. McLerran, A.I. Vainshtein and M.B. Voloshin, Electroweak interactions become strong at energy above approximately $10 \mathrm{TeV}$, Phys. Rev. D 42 (1990) 171 [INSPIRE].

[7] L.D. McLerran, A.I. Vainshtein and M.B. Voloshin, Strong instanton induced amplitudes in a weakly coupled theory, Phys. Rev. D 42 (1990) 180 [InSPIRE].

[8] M.P. Mattis, The riddle of high-energy baryon number violation, Phys. Rept. 214 (1992) 159 [INSPIRE].

[9] F.L. Bezrukov et al., Semiclassical study of baryon and lepton number violation in high-energy electroweak collisions, Phys. Rev. D 68 (2003) 036005 [hep-ph/0304180] [INSPIRE].

[10] F.R. Klinkhamer and N.S. Manton, A saddle point solution in the Weinberg-Salam theory, Phys. Rev. D 30 (1984) 2212 [INSPIRE].

[11] K. Funakubo, K. Fuyuto and E. Senaha, Does a band structure affect sphaleron processes?, arXiv:1612.05431 [INSPIRE].

[12] A. Ringwald, Electroweak instantons/sphalerons at VLHC?, Phys. Lett. B 555 (2003) 227 [hep-ph/0212099] [INSPIRE].

[13] A. Ringwald, An upper bound on the total cross-section for electroweak baryon number violation, JHEP 10 (2003) 008 [hep-ph/0307034] [INSPIRE].

[14] S.H.H. Tye and S.S.C. Wong, Baryon number violating scatterings in laboratories, Phys. Rev. D 96 (2017) 093004 [arXiv: 1710.07223] [INSPIRE].

[15] S.H.H. Tye and S.S.C. Wong, Bloch wave function for the periodic sphaleron potential and unsuppressed baryon and lepton number violating processes, Phys. Rev. D 92 (2015) 045005 [arXiv: 1505. 03690] [INSPIRE].

[16] J. Ellis and K. Sakurai, Search for sphalerons in proton-proton collisions, JHEP 04 (2016) 086 [arXiv: 1601.03654$]$ [INSPIRE]. 
[17] J. Ellis, K. Sakurai and M. Spannowsky, Search for sphalerons: IceCube vs. LHC, JHEP 05 (2016) 085 [arXiv : 1603.06573] [INSPIRE].

[18] G. Brooijmans, P. Schichtel and M. Spannowsky, Cosmic ray air showers from sphalerons, Phys. Lett. B 761 (2016) 213 [arXiv:1602.00647] [InSPIRE].

[19] M. Spannowsky and C. Tamarit, Sphalerons in composite and non-standard Higgs models, Phys. Rev. D 95 (2017) 015006 [arXiv:1611.05466] [InSPIRE].

[20] Y. Jho and S.C. Park, Constraining new physics with high multiplicity: I. Ultra-high energy cosmic rays on air-shower detector arrays, arXiv:1806.03063 [INSPIRE].

[21] D.G. Cerdeño, P. Reimitz, K. Sakurai and C. Tamarit, $B+L$ violation at colliders and new physics, JHEP 04 (2018) 076 [arXiv: 1801.03492] [INSPIRE].

[22] A. Ringwald, K. Sakurai and B.R. Webber, Limits on electroweak instanton-induced processes with multiple boson production, JHEP 11 (2018) 105 [arXiv:1809.10833] [INSPIRE].

[23] L.A. Anchordoqui and I. Antoniadis, Supersymmetric sphaleron configurations as the origin of the perplexing ANITA events, Phys. Lett. B 790 (2019) 578 [arXiv:1812.01520] [INSPIRE].

[24] A. Papaefstathiou and K. Sakurai, Determining the helicity structure of third generation resonances, JHEP 06 (2012) 069 [arXiv:1112.3956] [INSPIRE].

[25] M. Bahr et al., HERWIG++ physics and manual, Eur. Phys. J. C 58 (2008) 639 [arXiv:0803.0883] [INSPIRE].

[26] S. Gieseke et al., HERWIG++ 2.5 release note, arXiv:1102.1672 [INSPIRE].

[27] K. Arnold et al., HERWIG++ 2.6 release note, arXiv:1205.4902 [INSPIRE].

[28] J. Bellm et al., HERWIG++ 2.7 release note, arXiv:1310.6877 [INSPIRE].

[29] J. Bellm et al., HERWIG 7.0/HERWIG++ 3.0 release note, Eur. Phys. J. C 76 (2016) 196 [arXiv: 1512.01178] [INSPIRE].

[30] J. Bellm et al., HERWIG 7.1 release note, arXiv:1705.06919 [INSPIRE].

[31] M. Gibbs, A. Ringwald and F. Schrempp, QCD instanton induced final states in deep inelastic scattering, in the proceedings of the Deep inelastic scattering and QCD, April 24-28, Paris, France (1995), hep-ph/9506392 [INSPIRE].

[32] S. Moch, A. Ringwald and F. Schrempp, Instantons in deep inelastic scattering: The Simplest process, Nucl. Phys. B 507 (1997) 134 [hep-ph/9609445] [INSPIRE].

[33] A. Ringwald and F. Schrempp, QCDINS 2.0: a Monte Carlo generator for instanton induced processes in deep inelastic scattering, Comput. Phys. Commun. 132 (2000) 267 [hep-ph/9911516] [INSPIRE].

[34] C.M. Harris, P. Richardson and B.R. Webber, CHARYBDIS: a black hole event generator, JHEP 08 (2003) 033 [hep-ph/0307305] [INSPIRE].

[35] C.M. Harris et al., Exploring higher dimensional black holes at the large hadron collider, JHEP 05 (2005) 053 [hep-ph/0411022] [INSPIRE].

[36] J.A. Frost et al., Phenomenology of production and decay of spinning extra-dimensional black holes at hadron colliders, JHEP 10 (2009) 014 [arXiv:0904.0979] [INSPIRE].

[37] D.-C. Dai et al., BlackMax: a black-hole event generator with rotation, recoil, split branes and brane tension, Phys. Rev. D 77 (2008) 076007 [arXiv:0711.3012] [INSPIRE]. 
[38] V.V. Khoze and M. Spannowsky, Higgsplosion: solving the hierarchy problem via rapid decays of heavy states into multiple Higgs bosons, Nucl. Phys. B 926 (2018) 95 [arXiv: 1704.03447] [INSPIRE].

[39] P. Richardson, Simulations of R-parity violating SUSY models, Ph.D. thesis, Oxford University, Oxford U.K. (2000), hep-ph/0101105 [INSPIRE].

[40] R. Kleiss, W.J. Stirling and S.D. Ellis, A New Monte carlo treatment of multiparticle phase space at high-energies, Comput. Phys. Commun. 40 (1986) 359 [INSPIRE].

[41] S. Plätzer, RAMBO on diet, arXiv:1308.2922 [INSPIRE].

[42] R. Kleiss and W.J. Stirling, Massive multiplicities and Monte Carlo, Nucl. Phys. B 385 (1992) 413 [INSPIRE].

[43] V.V. Khoze and A. Ringwald, Total cross-section for anomalous fermion number violation via dispersion relation, Nucl. Phys. B 355 (1991) 351 [INSPIRE].

[44] CMS collaboration, Search for black holes and sphalerons in high-multiplicity final states in proton-proton collisions at $\sqrt{s}=13 \mathrm{TeV}$, JHEP 11 (2018) 042 [arXiv:1805.06013] [INSPIRE].

[45] M.J. Gibbs and B.R. Webber, HERBVI: a program for simulation of baryon and lepton number violating processes, Comput. Phys. Commun. 90 (1995) 369 [hep-ph/9504232] [INSPIRE].

[46] G.R. Farrar and R.-b. Meng, Baryon number violation in high-energy collisions, Phys. Rev. Lett. 65 (1990) 3377 [INSPIRE].

[47] M.J. Gibbs, A. Ringwald, B.R. Webber and J.T. Zadrozny, Monte Carlo simulation of baryon and lepton number violating processes at high-energies, Z. Phys. C 66 (1995) 285 [hep-ph/9406266] [INSPIRE].

[48] J. Butterworth et al., PDF4LHC recommendations for LHC Run II, J. Phys. G 43 (2016) 023001 [arXiv: 1510.03865] [INSPIRE].

[49] A. Papaefstathiou, K. Sakurai and S. Plaetzer, A Monte Carlo event generator for instanton/sphaleron processes in HERWIG, (2019).

[50] S. Ovyn, X. Rouby and V. Lemaitre, DELPHES, a framework for fast simulation of a generic collider experiment, arXiv:0903.2225 [INSPIRE].

[51] M. Selvaggi, DELPHES 3: a modular framework for fast-simulation of generic collider experiments, J. Phys. Conf. Ser. 523 (2014) 012033 [inSPIRE].

[52] M. Cacciari, G.P. Salam and G. Soyez, The anti-k $k_{t}$ jet clustering algorithm, JHEP 04 (2008) 063 [arXiv: 0802.1189] [INSPIRE].

[53] M. Cacciari, G.P. Salam and G. Soyez, FastJet user manual, Eur. Phys. J. C 72 (2012) 1896 [arXiv:1111.6097] [INSPIRE].

[54] M. Guzzi et al., CT10 parton distributions and other developments in the global QCD analysis, arXiv:1101.0561 [INSPIRE]. 